\title{
BMJ Open Self-efficacy assessment tools for individuals with depression: protocol for a systematic review of the literature
} Marina Araújo Rosas, ${ }^{1}$ Tattiana Dias de Carvalho Cordeiro, ${ }^{2}$
Tatiana de Paula Santana da Silva, ${ }^{3}$ Ada Salvetti Cavalcanti Caldas, ${ }^{3}$
Carlos Eduardo de Souza Leão Ribeiro, ${ }^{4}$ Manoel Henrique da Nóbrega Marinho, ${ }^{5}$
Murilo Duarte da Costa Lima, ${ }^{3}$ Selene Cordeiro Vasconcelos ${ }^{2}$
To cite: Rosas MA,

Cordeiro TDC, Silva TPS, et al. Self-efficacy assessment tools for individuals with depression: protocol for a systematic review of the literature. BMJ Open 2019;9:e021895. doi:10.1136/ bmjopen-2018-021895

- Prepublication history and additional material for this paper are available online. To view these files, please visit the journal online (http://dx.doi. org/10.1136/bmjopen-2018021895).

MAR, TDCC, TPSS, ASCC, CESLR, MHNM, MDCL and SCV contributed equally.

Received 25 January 2018 Revised 9 March 2019 Accepted 7 May 2019

Check for updates

(C) Author(s) (or their employer(s)) 2019. Re-use permitted under CC BY-NC. No commercial re-use. See rights and permissions. Published by BMJ.

For numbered affiliations see end of article.

Correspondence to

Prof Marina Araújo Rosas; marinaarosas@gmail.com

\section{ABSTRACT}

Introduction Depression is a common debilitating disease that affects individuals in all age groups. The impact of the diagnosis extends beyond the individual, with negative effects on mental health, physical health and social well-being. Self-efficacy has been referenced as an important aspect to the prognosis of mood disorders by conferring co-responsibility to the affected individual to face his/her health problems. Several assessment tools are found in the literature for measuring self-efficacy, but it is not yet clear which of these measures are more applicable to individuals with mood disorders, particularly depression. Thus, the aim of present study is to propose a systematic review to examine the psychometric properties and applicability of assessment tools designed to measure self-efficacy in individuals with symptoms and/or a diagnosis of depression.

Methods and analysis This protocol is reported in accordance with the Preferred Reporting Items for Systematic Review and Meta-Analysis Protocols statement and the review will be reported in accordance with the Preferred Reporting Items for Systematic Reviews and Meta-Analyses statement. The evaluation of the psychometric properties of the health outcome measures will be conducted according to COSMIN guidelines. Two independent reviewers will perform the electronic searches in the PubMed, Web of Science, Psyclnfo, SCOPUS and CINAHL databases, followed by the use of the 'snowball' strategy. The inclusion criteria will be (1) instrument validation studies, (2) developed with individuals of any age (3) with symptoms or a diagnosis of depression. Two independent reviewers will analyse the titles and abstracts of the articles retrieved during the search for pre-selection, followed by full-text analyses to determine inclusion in the review based on the eligibility criteria. Cases of a divergence of opinion will be resolved by a third reviewer. Descriptive analysis of the articles will be performed (data on participants, characteristics, psychometric properties and clinical usefulness of the assessment tools).

Ethics and dissemination The proposed systematic review will provide information on assessment tools employed to measure self-efficacy with regard to coping with depression, offering data on the psychometric properties, strong and weak points, and clinical applicability. As a secondary analysis of the literature, the approval of an ethics committee is not required.
Strengths and limitations of this study

- No previous systematic reviews addressing to analyse assessment tools designed to measure self-efficacy for coping with depression this issue was found in the literature.

- Instruments will be presented with details of their applicability according to population and age group.

- The proposed review strategy it will include six important health databases, with no restrictions imposed regarding language or year of publication.

- The intention is to present the best instruments to enable decision-making and the development of therapeutic guidelines for coping with depression.

- The limitations may be related to the different composition of the samples in the articles selected for the review.

\section{PROSPERO registration number CRD42017078707}

\section{BACKGROUND}

Depression is a mood disorder ${ }^{1}$ and one of the main causes of disability, affecting more than 300 million individuals throughout the world. ${ }^{2}$ It is estimated that more than $9 \%$ of the population is affected in some countries. ${ }^{3}$ Socioeconomic factors are related to depression, as this disorder compromises social functioning and generates high costs to both the individual and society in terms of expenditure on clinical care and the loss of productivity. ${ }^{3-5}$

The psychopathology of depression involves a complex set of factors that compromise physical function and mechanisms of self-regulation, with a significant impact on the behavioural dynamics of the affected individual. ${ }^{6}$ One of the mechanisms of self-regulation is denominated self-efficacy, which is conceived as a belief or trust in one's own capacity to organise and execute a specific behaviour necessary to the attainment of a particular goal. ${ }^{78}$ Thus, self-efficacy is a proactive stance ${ }^{9}$ driving one 
to perform actions in one's own favour to cope with difficulties and ensure well-being. ${ }^{9}$ Greater self-confidence in one's ability to perform a specific behaviour has a positive effect on coping with an adverse situation, such as depression, and therefore exerts an influence on the prognosis and treatment. $^{10}$

For example, McCusker $e t a l^{11}$ found that self-efficacy was consistently correlated with health promotion and the maintenance of health behaviours in depressed patients. ${ }^{11}$ Depressed individuals often disengage from pleasurable or challenging activities. Therefore, they miss out on opportunities to exercise their skills and maintain their sense of self-efficacy. ${ }^{12}$ The loss of belief in one's skills further perpetuates the disengagement from activities and increases the severity of the depressive symptoms. ${ }^{12}$ Thus, the re-establishment of self-efficacy could play an important role in the return of functional abilities and the measurement of this construct therefore has implications for therapeutic interventions aimed at increasing the involvement of depressed individuals in such activities. ${ }^{12}$

The use of valid, reliable assessment tools for measuring self-efficacy in individuals with depression could contribute to the planning and implementation of intervention strategies aimed at enhancing the self-regulation of mood and managing situations imposed by the health-illness process related to depression. Thus, the guiding question for the study proposed in the present protocol is 'Do assessment tools employed to measure self-efficacy in individuals with depression have adequate psychometric properties?'

\section{OBJECTIVES}

The aim of the present study is to propose a systematic review of the literature to analyse the psychometric properties of assessment tools employed to measure self-efficacy in individuals with depression.

\section{METHOD/DESIGN}

\section{Design and registration of the study}

This systematic review was registered with the International Prospective Registry of Systematic Reviews (http:// www.crd.york.ac.uk/PROSPERO/) on 22 November 2017. The protocol was written and reported using the Preferred Reporting Items for Systematic Review and Meta-Analysis Protocols statement ${ }^{13}$ (see online supplementary file 1) and the systematic review will be developed based on the Preferred Reporting Items for Systematic Reviews and Meta-Analyses (PRISMA) statement ${ }^{14}$ (see online supplementary file 2).

\section{Search strategy}

To answer the guiding question of the study, searches will be performed in the PubMed, Web of Science, PsycInfo, Cochrane, SCOPUS and CINAHL databases. The 'snowball' method will be employed to broaden the search, which involves hand searches of the reference lists of the selected studies for the identification of potentially eligible studies not retrieved from the databases. ${ }^{14}$ The following search terms will be used: (1) depression, (2) self-efficacy, (3) validation studies, (4) assessment tools and (5) psychometric properties. The detailed search strategy for the PubMed database can be found in online supplementary file 3 .

\section{Inclusion of articles}

The PRISMA guidelines ${ }^{14}$ will be followed, with the creation of a flowchart illustrating the article selection process.

The proposed review will include original articles conducted for the development of assessment tools to measure self-efficacy (the belief or expectation that a particular task can be achieved and the expected result can be attained through personal effort ${ }^{78}$ in individuals with depressive symptoms or a diagnosis of depression and describe the psychometric properties as well as the validation process of the assessment tool. The articles must describe that depressive symptoms or the diagnosis of depression were defined by a psychiatrist (based on the criteria of the Diagnostic and Statistical Manual of Mental Disorders, ${ }^{1}$ in its fifth edition or detected through a validated instrument). Due to the specificity of the simultaneous management of self-efficacy and depression, the decision was made not to specify the age range of the target population used in the development of evaluation tools. The systematic review article will present an analysis of each assessment tool.

All assessment tools that measure self-efficacy in depressed individuals will be eligible, independently of the number of validated psychometric properties and type of assessment tool (scale, questionnaire or other tool developed to measure this phenomenon). No restrictions will be imposed regarding language or year of publication. Articles will be selected based on the criteria listed in table 1 .

\section{Exclusion criteria}

Literature reviews with any methodological design and articles that do not present the psychometric properties of the assessment tool employed and/or its validation process will be excluded.

\section{Screening, data extraction and content analysis}

Articles duplicated in the databases will only be counted once. In studies with the absence of pertinent data, the authors will be contacted for the acquisition of additional information.

Two independent reviewers (CESLR/MHNM) will perform the searches of the databases for pertinent articles and another two independent researchers (MAR/ ASCC) will perform the selection of articles for the systematic review. Analyses of the titles and abstracts will be performed for the pre-selection of potentially eligible studies. Pre-selected articles will be submitted to full-text analysis based on the eligibility criteria for the determination of those to be included in the review. In cases of a divergence of opinion between the reviewers, a third reviewer (SCV) will conduct the discussion until a consensus is reached. 
Table 1 Inclusion criteria for systematic review

Individuals of any age with depressive symptoms or a diagnosis of depression attested by a psychiatrist or based on the fifth edition of the Diagnostic and Statistical Manual of Mental Disorders ${ }^{1}$ or

Population detected by a validated instrument.

$\begin{array}{ll}\text { Intervention/exposure } & \begin{array}{l}\text { Administration of assessment tool to } \\ \text { measure self-efficacy. }\end{array} \\ \text { Comparison } & \begin{array}{l}\text { The comparator was not used because } \\ \text { there is no assessment tool considered } \\ \text { the gold standard for measuring self- } \\ \text { efficacy with regard to coping with } \\ \text { depression. A standard gold tool will be } \\ \text { suggested based on the findings of the } \\ \text { proposed systematic review. }\end{array}\end{array}$

$\begin{array}{ll}\text { Outcome } & \begin{array}{l}\text { Analysis of the psychometric properties } \\ \text { of the assessment tools for measuring } \\ \text { self-efficacy with regard to coping } \\ \text { with depression and the possibility of } \\ \text { indicating a gold-standard assessment } \\ \text { tool for this purpose. }\end{array} \\ \text { Study design } & \begin{array}{l}\text { Methodological studies for the validation } \\ \text { of assessment tools for measuring } \\ \text { self-efficacy with regard to coping with } \\ \text { depression. }\end{array}\end{array}$

Data extraction will include, but not be limited to, the characteristics of the study (country, year of publication, journal in which the article was published, study design and period of study), population (description of study population, eligibility criteria, sample size), intervention (assessment tool employed) and measure of self-efficacy (expectation of efficacy/outcomes/scores, quality of the study and conclusions). During the data extraction process, the chart will have space available for the inclusion of additional items, as necessary (see online supplementary file 4).

\section{Appraisal of methodological quality of studies included in review}

The COSMIN Risk of Bias checklist ${ }^{15}$ will be used to determine the methodological quality of the studies included in the systematic review. This list has nine topics: internal consistency, reliability, measurement error, content validity, construct validity (structural validity, hypothesis tests and cross-cultural validity), criterion validity and responsiveness. ${ }^{16-20}$ Each item will be scored on a four-point classification scale for the determination of methodological quality: excellent, good, fair or poor. For example, if an item in the reliability box is scored as poor, the methodological quality in terms of reliability is classified as poor. A poor score on any item is considered a fatal flaw. ${ }^{20}$

Two independent reviewers (MAR/ASCC) will appraise each study. In cases of a divergence of opinion between the reviewers, a third reviewer (SCV) will conduct the discussion until a consensus is reached.

\section{Evaluation of clinical usefulness of assessment tools}

The evaluation criteria for interpretability and clinical usefulness (viability) are listed below ${ }^{21}$ :
- Total time required for the administration, analysis and interpretation of the data obtained using the assessment tool: $<10 \mathrm{~min}$ (three points); $10-30 \mathrm{~min}$ (two points); 30-60 $\min$ (one point) and $>1$ hour $(0$ points).

- Cost of assessment tool: $<£ 100$ (3 points); £100-£500 (two points); £500-£1000 (one point); $£ 1000$ (zero).

- Need for specialised equipment and training for use: none (two points); yes, but simple and clinically viable (one point); yes and not clinically viable/unknown (zero).

- Portability of tool (can it be taken to the patient?): yes, easily (fits in pocket) (two points); yes (fits in a carrying case) (one point); no or very difficult (zero).

- Accessibility of tool (are detailed instructions for use available?): yes (complete operating procedure/ instruction manual can be obtained in article or site) (two points); no, but the operation can be performed simply based on the description in the article (one point); no available instructions for use (zero).

The assessment tools analysed in the proposed systematic review will be printed on paper and self-reports of individuals will be used to measure self-efficacy. Therefore, no specialised equipment will be necessary and all materials will be easy to transport. However, to ensure the validity of the evaluation based on the criteria listed above, the portability criterion will receive two points and the need for training will be scored as follows: none (two points); yes, but simple and clinically viable (one point); yes and not clinically viable/unknown (zero), with the removal of the need for specialised equipment. The use of these criteria will result in a maximum of 12 points and assessment tools that receive a score of less than 10 points will be considered not recommendable for clinical practice.

\section{Data synthesis}

A systematic narrative synthesis will be drafted in the form of texts and explanatory charts on the main findings of the analysis of the assessment tools included in the final sample. The psychometric quality of the assessment tools will also be evaluated. The results of the methodological and psychometric quality appraisal will be combined to reach a synthesis of the best level of evidence for each assessment tool.

The entire development process for the protocol article and systematic review will be stored in both electronic form and hard copies (printed on paper) through to the publication of the respective manuscripts to ensure accessibility to the data and enable clarifications for the referees during the peer-review process.

An overview of all the assessment tools analysed will be presented specifying the validated psychometric properties to enable the recommendation of a single assessment tool that could be considered the gold standard for measuring self-efficacy in depressed individuals.

The expected primary outcome is to contribute to the clinical management of depressed patients by recommending a gold standard assessment tool for measuring 
self-efficacy in individuals with depression. Secondary outcomes are related to the production of robust scientific evidence capable of contributing to the decision-making process in clinical practice regarding the improvement of self-efficacy as a mechanism for coping with depression and promoting the wider use of the evaluation instrument recommended in practice to guide interventions and evaluate their effects.

\section{Patient and public involvement}

The proposed study is a literature review and will therefore not have the direct involvement of patients.

\section{Ethics and dissemination}

The proposed systematic review will provide information on the assessment tools used to measure self-efficacy with regard to coping with depression. It will offer information on the psychometric properties of these assessment tools, with an emphasis on the strong and weak points as well as the clinical applicability.

The choice of validated measures can significantly contribute to the understanding of the mechanism of self-regulation of individuals with depression. Such assessment tools can be effective at directing therapy and adopting strategic measures for new cases.

Like any systematic review, the proposed study could exhibit selection bias for not including articles that have not yet been published (those in print). This decision on the part of the authors was made due to the possible difficulty in achieving permission to use manuscripts from the authors of articles that are in the publication phase. Bias will be minimised by the rigorous selection of the final sample of articles based on the eligibility criteria and the use of a search strategy involving independent reviewers in each step of the article selection process, with the resolution of conflicts by a third reviewer.

The findings will be divulged in peer-reviewed journals and will include all complementary material, with the detailing of the search strategy as well as the extraction, compilation and analysis of the data. As a systematic review is proposed, ethical approval is not required. The protocol and systematic review compose activities of the doctoral programme in neuropsychiatry and behavioural sciences of the Federal University of Pernambuco, Brazil.

\section{Author affiliations}

${ }^{1}$ Department of Occupational Therapy and Department of Neuropsychiatry, Federal University of Pernambuco, Recife, PE, Brazil

${ }^{2}$ Department of Nursing in Public Health, Federal University of Paraíba, JoãoPessoa, PB, Brazil

${ }^{3}$ Department of Neuropsychiatry, Federal University of Pernambuco, Recife, PE, Brazil

${ }^{4}$ Federal University of Pernambuco, Recife, PE, Brazil

${ }^{5}$ University of Pernambuco, Recife, PE, Brazil

Contributors All authors contributed substantially to the design and development of the study and participated in the elaboration of the request for submission. MAR, TDCC, TPSS, ASCC, CESLR, SCV conceived the study, developed the criteria, carried out the search and selection of the studies and wrote the present systematic review protocol article. MHNM, SCV, MDCL guided all phases of this systematic review protocol article and performed a critical review of the manuscript. All authors read and approved the final version.

Funding The authors have not declared a specific grant for this research from any funding agency in the public, commercial or not-for-profit sectors.

Competing interests None declared.

Patient consent for publication Not required.

Provenance and peer review Not commissioned; externally peer reviewed.

Open access This is an open access article distributed in accordance with the Creative Commons Attribution Non Commercial (CC BY-NC 4.0) license, which permits others to distribute, remix, adapt, build upon this work non-commercially, and license their derivative works on different terms, provided the original work is properly cited, appropriate credit is given, any changes made indicated, and the use is non-commercial. See: http://creativecommons.org/licenses/by-nc/4.0/.

\section{REFERENCES}

1. American Psychiatric Association. DSM-5: Manual diagnóstico e estatístico de transtornos mentais. Artmed Editora 2014:155-89.

2. World Health Organization. Depression and other common mental disorders: global health estimates. Geneva: World Health Organization, 2017.

3. Centers for Disease Control and Prevention (CDC). Current depression among adults---United States, 2006 and 2008. MMWR Morb Mortal Wkly Rep 2010;59:1229-35.

4. Hawton K, Casañas I Comabella C, Haw C, et al. Risk factors for suicide in individuals with depression: a systematic review. J Affect Disord 2013;147:17-28.

5. McCrone PR, Dhanasiri S, Patel A, et al. Paying the price: the cost of mental health care in England to 2026. London: King's Fund 2008.

6. Nóbrega LG, Britto I. Evaluation and treatment of problem behavior of two people with depression. Rev Bras de Terapia Comportamental e Cognitiva 2017;19:128-45.

7. Bandura A. Self-efficacy: toward a unifying theory of behavioral change. Psychol Rev 1977;84:191-215.

8. Bandura A. A sociocognitive analysis of substance abuse: an agentic perspective. Psychol Sci 1999;10:214-7.

9. Litt MD. Self-efficacy and perceived control: cognitive mediators of pain tolerance. J Pers Soc Psychol 1988;54:149-60.

10. Suzanne P. Development of the Depression Coping Self-Efficacy Scale (DCSES). Arch. Psychiatr Nur 2000;14:276-84.

11. McCusker J, Lambert SD, Cole MG, et al. Activation and self-efficacy in a randomized trial of a depression self-care intervention. Health Educ Behav 2016;43:716-25.

12. Milanovic M, Ayukawa E, Usyatynsky A, et al. Self Efficacy in depression: bridging the gap between competence and real world functioning. J Nerv Ment Dis 2018;206:350-5.

13. Moher D, Shamseer L, Clarke M, et al. Preferred reporting items for systematic review and meta-analysis protocols (PRISMA-P) 2015 statement. Syst Rev 2015;4:1.

14. Liberati A, Altman DG, Tetzlaff J, et al. The PRISMA statement for reporting systematic reviews and meta-analyses of studies that evaluate health care interventions: explanation and elaboration. PLoS Med 2009;6:64-94.

15. Mokkink LB, Prinsen CA, Bouter LM, et al. The COnsensus-based Standards for the selection of health Measurement INstruments (COSMIN) and how to select an outcome measurement instrument. Braz J Phys Ther 2016;20:105-13.

16. Ridley D. The literature review: a step-by-step guide for students. London: Sage, 2008.

17. Mokkink LB, de Vet HCW, Prinsen CAC, et al. COSMIN Risk of Bias checklist for systematic reviews of patient-reported outcome measures. Qual Life Res 2018;27:1171-9.

18. Mokkink LB, Terwee CB, Gibbons E, et al. Inter-rater agreement and reliability of the COSMIN (COnsensus-based Standards for the selection of health status Measurement Instruments) checklist. BMC Med Res Methodol 2010;10:82.

19. Mokkink LB, Terwee CB, Stratford PW, et al. Evaluation of the methodological quality of systematic reviews of health status measurement instruments. Qual Life Res 2009;18:313-33.

20. Terwee CB, Mokkink LB, Knol DL, et al. Rating the methodological quality in systematic reviews of studies on measurement properties: a scoring system for the COSMIN checklist. Qual Life Res 2012;21:651-7.

21. Tyson SF, Brown P. How to measure fatigue in neurological conditions? A systematic review of psychometric properties and clinical utility of measures used so far. Clin Rehabil 2014;28:804-16. 